\title{
IDENTIFYING SUITABLE AREAS FOR SMALL FARM RESERVOIR IN AGUSAN DEL NORTE USING GEOGRAPHIC INFORMATION SYSTEM
}

\author{
Antonietto O. Cacayan Jr², Arnold G. Apdohan ${ }^{12}$, Aljon E. Bocobo ${ }^{2}$, Jerald L. Ruta ${ }^{2}$ \\ ${ }^{1}$ College of Engineering and Geosciences, Caraga State University, Ampayon, Butuan City, Philippines - ceit.carsu.edu.ph \\ ${ }^{2}$ Center for Resource Assessment, Analytics, and Emerging Technologies, CSU, Ampayon, Butuan City - \\ antoncacayan@gmail.com
}

\section{Commission IV}

KEY WORDS: Geographic Information System, Small Farm Reservoir, rainfall harvesting, irrigation, suitability mapping

\begin{abstract}
:
The Philippines, as a part of Southeast Asia, falls into the zone categorized with agricultural areas prone to drought. Mitigating the concerns due to such vulnerability and also catering the needs of small-time farmers, rainfall harvesting with the use of agricultural structures called Small Farm Reservoirs (SFRs) used for storing water for irrigation are being used and deployed. This study aims to identify ideal locations for SFR which is essential due to the concerns of its geographical appropriateness and its vulnerability to environmental and other concerns such as seepage, sustainability, and optimizing yield. Geographic Information System through the ArcMap software is a modern tool for decision making, especially in the concerns of suitability and vulnerability assessment and was used in creating the suitability map for SFR in this study. The suitability map was created with the use of pre-processed raster data sets for precipitation, soil texture, slope, irrigation status. Weights for the weighted overlay were acquired from the standardized protocol for Small Scale Irrigation Project (SSIP) standardized protocol for site suitability identification from the Department of Agriculture - Bureau of Soil and Water Management (DA-BSWM) Philippines. Existing SFRs in the study area were validated through site visitation and were evaluated for usability and performance. The suitability map created accurately identified suitable zones for establishing SFRs with a result of 7 out of 7 existing SFRs are within the suitable zones, six (6) sites in the moderately suitable and one (1) on marginally suitable
\end{abstract}

\section{INTRODUCTION}

Agriculture which has been one of the significant pillars of Philippine economy, food supply and living is into the risk due to increase in urbanization and also with climate change such as drought and flooding (Redfern et al. 2015). Conventional irrigation is already practised to address the existent problem on the supply of water to agricultural areas but due to the constraints such as topography, soil drainage, water storage problem and the ratio of the size of irrigation facility to potential service area leaves a significant number of farmers to rely on natural irrigation classified as rainfed areas. (Bhuiyan and Zeigler 2018 ). The Philippines, as a part of Southeast Asia, falls into the zone categorized with $58 \%$ of rainfed agricultural areas as drought-prone (Garrity et al. 1986). To mitigate the concerns of the vulnerability of rainfed agricultural areas and to cater the needs of individual farmers especially those with small farms, rainfall harvesting in the form of On-Farm Reservoirs (OFR) or Small Farm Reservoirs (SFR) were used. These structures are to be used for water embankment during rainfall for controlled irrigation and avoid flooding during the rainy season and would serve as water storage during the dry season (Ines et al. 2018). Geographic Information System (GIS) has already been used on several occasions, especially in the geographic concerns on the field of agriculture. The capability of the GIS technology to mathematically consolidate data inputs to come up with visual outputs which are to be used which are highly influential in decision making, especially in site selection and vulnerability assessment. Agusan del Norte as an area with vast agricultural land and limited irrigation facilities has been selected for the conduct of this study. The objectives of this study are to (1) create a suitability map for Small Farm Reservoirs, (2) locate and validate existing small farm reservoirs in Agusan del Norte and (3) assess the accuracy of the generated suitability map through overlaying validated existing SFRs.

\section{MATERIALS AND METHODS}

\subsection{Site Selection.}

The area is Agusan del Norte, Caraga Region, Philippines lo-cated at $\left(8.9456^{\circ} \mathrm{N}, 125.5319^{\circ} \mathrm{E}\right)$ with a total land area of $2,730.24 \mathrm{~km} 2$.

\subsection{Rainfall Map}

Generated through the Spline Interpolation Method using the Climatologic Precipitation Normals from Philippine Atmospheric Geophysical and Astronomical Services Administration (PAGASA) and reclassified through the Reclassify Tool in ArcMap

\subsection{Soil Map}

The Soil map raster data set was acquired from the Bureau of Soil and Water Management (BSWM) and also reclassified according to soil texture.

\subsection{Slope Map}

Slope Map was generated through the use of the Digital Eleva-tion Model (DEM) (5m resolution) and Slope Tool and Reclas-sify Tool in ArcMap. 


\subsection{Potential Service Area Map}

Generated through intersecting with the use of Intersect Tool in ArcMap of the Land Use/Land Cover Map (selecting only the agricultural areas) and Slope Map then subtracting or excluding all currently irrigated areas with the use of Cut Tool in ArcMap.

\subsection{Validated Points}

Validated Points are geographic coordinates acquired through actual field validation survey with use of GPS.

\subsection{Weighted Overlay}

All maps were overlaid using the Weighted Overlay Tool in ArcMap to generate the suitability map for SFR. The weights for overlay were acquired and standardized protocol according to the standard set by the BSWM, National Water Resources Board, and all 13 participating State Universities and Colleges around the Philippines

\begin{tabular}{|c|c|c|c|}
\hline Factor & Description & $\begin{array}{c}\text { Suitability } \\
\text { Scale }\end{array}$ & Weight \\
\hline Average & $<1000 \mathrm{~mm}$ & 1 & \\
Annual & $1000-1200 \mathrm{~mm}$ & 2 & 40 \\
Rainfall & $>1200 \mathrm{~mm}$ & 3 & \\
& $\begin{array}{c}\text { Others (sand, silt } \\
\text { loam, silt, clay, }\end{array}$ & 0 & \\
& Mountain soil) & & \multirow{5}{*}{30} \\
Sandy loam & 1 & \\
& Sandy clay loam & 2 & \\
& Clay loam and & 3 & \\
silty clay loam & $-3^{\circ}$ & 1 & \\
Slope & $3-8^{\circ}$ & 2 & 30 \\
& $8-18^{\circ}$ & 3 & \\
Irrigation & $>18^{\circ}$ & 0 & \\
Status & Irrigated & 0 & Must \\
& Non-irrigated & 3 & \\
\hline
\end{tabular}

Table 1: Standardized weight distribution for SFR suitability map

\section{RESULTS AND DISCUSSION}

\subsection{Generated Small Farm Reservoir Suitability Map}

The generated map shows the areas that are highly, moderately, marginally and not suitable. It shows that majority of these suitable areas are at the flat areas of Agusan del Norte spanning from the lower parts of Buenavista, low lying areas of Butuan City and Cabadbaran and extends to the plain areas of Tubay, Santiago and some parts in Kitcharao. 6 out of 7 existing SFRs in Agusan del Norte reside inside the Moderate, and one on the marginally Suitable area and all are operational and functional. The map generated shows high to almost perfect accuracy since there was not any existing SFR that resides outside the suitable areas. The map suggests vast areas with considerable suitability of SFR establishment throughout the Agusan del Norte area. The location of suitable areas for SFR establishment also lies majorly on agricultural areas, specifically the rice areas of Agusan del Norte where the necessity irrigation is necessary and fundamental.

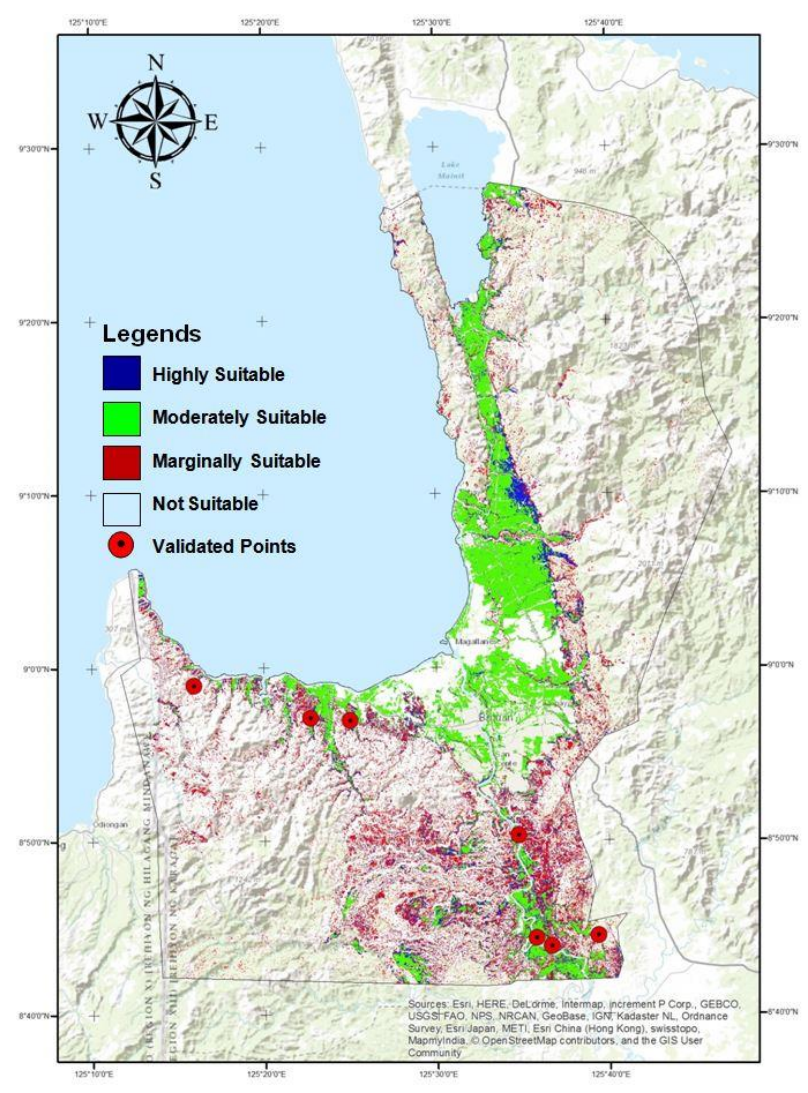

Figure 1. The generated SFR Suitability Map for Agusan del Norte

\section{CONCLUSION}

To address the vulnerability of drought and flood propensity of Agusan del Norte, the establishment of SFRs for rainfall harvesting is encouraged. Selection of suitable areas through analytical GIS-Based water resources assessment with use of factors considered in the actual selection yielded to an exceptional accuracy considering every functional and operational SFRs throughout the study area as validation. With this, it could be concluded that Agusan del Norte has a high potential of future SFR establishment and the methods and factors used for the data analysis is appropriate and has high accuracy, and the output could then be considered for future plans and decision making regarding the future irrigation projects.

\section{ACKNOWLEDGEMENT}

This research was made possible by the financial support of the Department of Agriculture - Bureau of Agricultural Research (DA-BAR), with the aid of Bureau of Soil and Water Management (BSWM) with collaboration of the Department of Agriculture - Caraga (DA-Caraga), National Irrigation Authority-Caraga (NIA-Caraga). 


\section{REFERENCES}

Redfern, S. K., Azzu, N., Binamira, J. S. (2015). Rice in Southeast Asia: Facing Risks and Vulnerabilities to Respond to Climate Change. (p 1-14). FAO

Garrity, D. P., Oldeman, L. R., Morris, R. A., Lenka, D. (1980) Rainfed lowland rice ecosystems: characterization and distribution. (pp 3-23) in Progres in rainfed lowland rice. International Rice Research Institute
Bhuiyan, S. I., Zeigler, R. S. (2018). On-farm rainwater storage and conservation system for drought alleviation: issues and challenges, (pp 1-6) in On-Farm Reservoir Systems for Rainfed Ricelands. International Rice Research Institute

Ines, R. L., Tuazon, J. B., Daag, M. N. (2018). Utilization of Small Farm Reservoir (SFR) for Upland Agriculture of Bataan, Philippines, (pp 1-6) International Journal of Applied Agricultural Sciences

\section{APPENDIX}

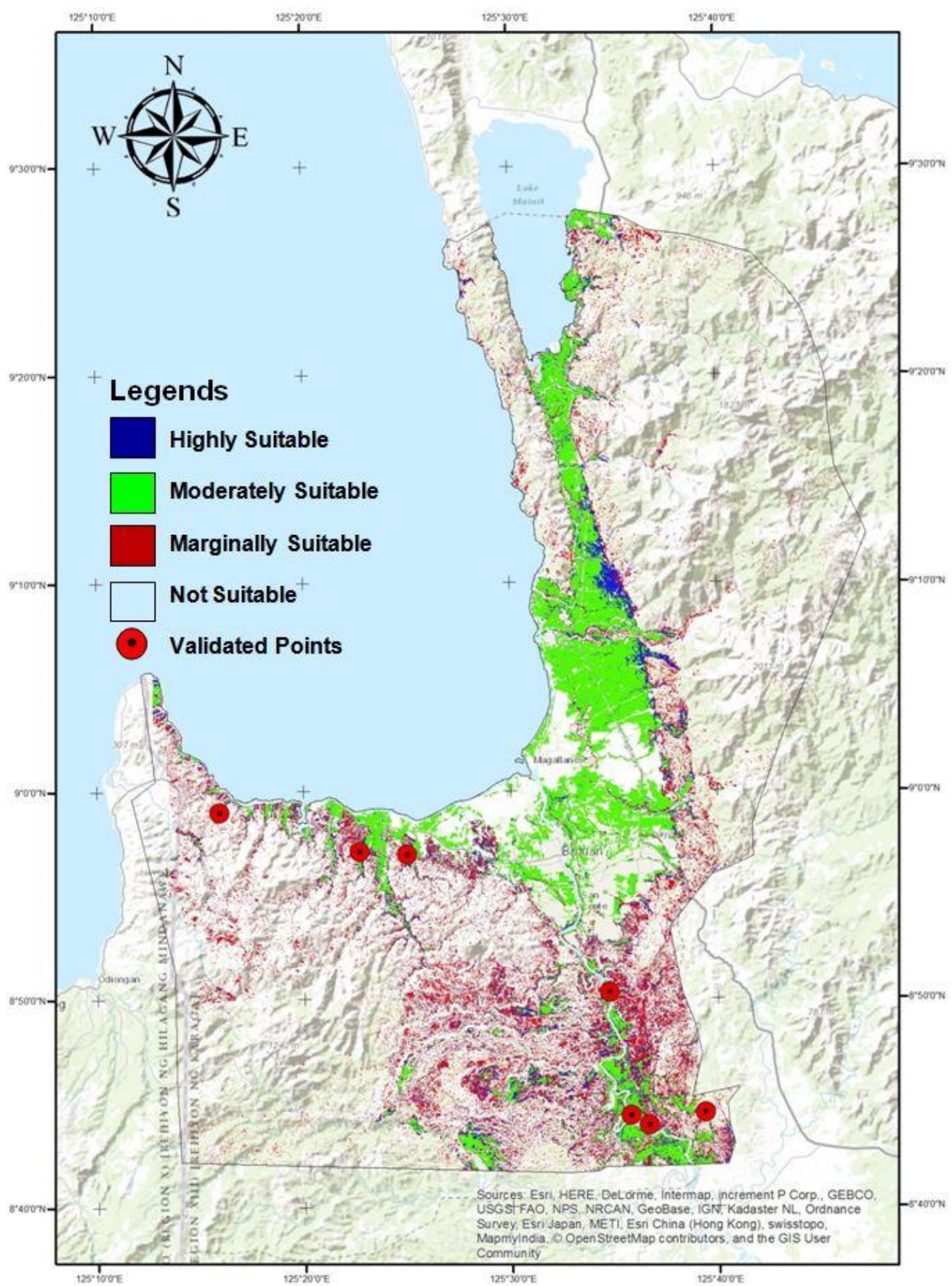

